\title{
Metodologia de Coleta de Insetos Aquáticos em Paredões de Cachoeiras
}

\author{
Helena Soares Ramos Cabette ${ }^{\bowtie}$ \& Lourivaldo Amancio de Castro
}

Universidade do Estado de Mato Grosso, Campus de Nova Xavantina, e-mail: hcabette@uol.com.br (Autor para correspondência ${ }^{\bowtie}$ ), louriamancio@yahoo.com.br.

EntomoBrasilis 7 (2): 161-163 (2014)

Resumo. Apresentamos uma nova metodologia de coleta de insetos aquáticos para ambientes de cachoeira, juntamente com a elaboração de um equipamento de amostragem. Propomos uma modificação do formato do rapiché ou coador tradicional, que com auxílio de uma trincha e espátula, possibilita a raspagem dos paredões, conseguindo retirar inclusive espécies de hábito agarrador. Propõe, ainda, adequação na metodologia de transecções de áreas fixas para a obtenção vertical de amostras comparável a transecções horizontais em riachos.

Palavras-Chave: Captura de insetos; Larvas; Macroinvertebrados; Riachos.

\section{Methods for Sampling Aquatic Insects on Walls of Waterfalls}

Abstract. We present a new method for sampling aquatic insects in waterfall environments, and the development of a sampling equipment. We propose a change in the shape of the dipnet or traditional hand sieve. This change in shape, with the addition of a brush and spatula, enables the scraping of walls, so that even species of grabber habits are sampled. We propose, also, an adaptation of the method of fixed area transects to obtain vertical samples similar to the obtained through horizontal transects in streams.

Keywords: Insect capture; Larvae; Macroinvertebrates; Streams.

$\mathbb{N}$ os processos de estabelecer planos de manejo em áreas dotadas de beleza cênica, as cachoeiras são elementos chave, mas trabalhos que discutem planos de manejos raramente elegem insetos como base para avaliação de alterações ou impactos. Insetos aquáticos são excelentes indicadores de qualidade ambiental, especialmente da água dos córregos ou rios e podem indicar, desde alterações severas (Nessimian et al. 2008; Silva-Pinto et al. 2012), ou mesmo serem excelentes indicadores de áreas bem conservadas, com fidelidade e especificidade (DIASSilva et al. 2010; Souza et al. 2011; Pereira et al. 2012).

Os métodos mais comuns para coleta de insetos aquáticos incluem coletas manuais, uso de redes de arrasto de diferentes formatos, rapichés e dragas (MERRITT et al. 1984; ROLDÁN-PÉREZ 1988), em diferentes desenhos amostrais, mas não incluem amostradores adequados a paredões de cachoeiras ou desenho amostral que permita comparações com trechos de menor declive.

O presente trabalho buscou estabelecer uma metodologia de coleta em que os dados obtidos em cachoeiras sejam comparáveis àqueles obtidos em transecções lineares traçadas a montante e jusante nos córregos, definindo subunidades (pseudoréplicas) que permitam análises ecológicas das comunidades, como por exemplo, a estimativa de riqueza de espécies por cada córrego. $\mathrm{O}$ modelo de transecção para paredões de cachoeiras foi modificado do proposto por De MARCo (1998) para inventários rápidos de odonatos adultos. O coletor, por sua vez, se adequa ao tamanho dos rapichés ( $18 \mathrm{~cm}$ de aro) utilizados em inventários em córregos do leste mato-grossense (САВETTE et al. 2010).

O coletor (Figura 1) apresenta aro em forma de D (21,5 x 19,5 $\mathrm{cm}$, Figura 1D), com o lado reto com uma aba fixada na borda em ângulo de $45^{\circ}$ (19 cm, detalhe no lado direito da Figura 1B), para permitir melhor ajuste do coador no paredão e direcionar a água para o interior do puçá. E em toda a volta do aro há uma ranhura mediana (o,8 $\mathrm{cm}$ de largura $\mathrm{x} 0,2 \mathrm{~cm}$ de profundidade) em baixo relevo (Figura 1B), para receber o punho com cordão da rede. $\mathrm{O}$ aro é ligado a um cabo de $27 \mathrm{~cm}$, fixado com rebites (Figura 1A).

As redes recambiáveis são confeccionadas em tela de nylon (malha de $0,5 \mathrm{~mm}$, destacado na Figura $1 \mathrm{C}$, à esquerda) com punho em tecido de algodão maleável (percal), por onde passa um cordão para ajuste com presilhas de plástico de pressão interna (similar àquelas usadas em mochilas, Figura $1 \mathrm{C}$, à direita). As redes tem apenas uma pequena sobra de largura em relação à circunferência do aro e são recambiáveis.

O Cinturão (Figura 2), que acompanha o coletor possui um reforço central para apoio do cabo da rede (Figura 2A). Compõem o conjunto de coleta anexada ao cinturão: trincha larga de pelo macio de 1,4" de largura (Figura 2B), uma espátula de pedreiro pequena (Figura 2C), o coletor propriamente dito (Figura 2D), pequenas presilhas (mini mosquetões $2 \mathrm{E}$ ) e redes sobressalentes (Figura 2F). A espátula e a trincha servem para escovar os espécimes aderidos na vegetação ou no paredão da cachoeira.

Todas as coletas se dão com uso de técnica de rapel e o uso do cinturão auxilia na liberação das mãos durante a amostragem, conforme o coletor termina uma subunidade da transecção, retira a rede, a fecha com o próprio cordão, e a substitui por uma nova para o próximo segmento. O material coletado nas redes, 
T após triagem em bandejas brancas, é fixado em álcool etílico a $85 \%$ (larvas e adultos).

O desenho amostral: realizou-se em uma transecção de $100 \mathrm{~m}$ lineares, subdivididos de 5 a $5 \mathrm{~m}$, totalizando 20 subunidades (De Marco 1998; CABette et al. 2010), o material coletado em cada subunidade (3 coadores ou rede) corresponde a uma subamostra, tomando o local como amostra e definindo como réplicas as coletas periódicas ao longo do tempo. Manteve-se a mesma metodologia acima para os trechos a montante e jusante da cachoeira, e estabeleceu-se seis divisões na transecção (no sentido vertical), com três unidades seriadas cada (sentido horizontal), totalizando 18 trechos ou subunidades distribuídos ao longo do paredão (Figura 3). No processo de coleta, em cada sessão são tomadas amostras no ambiente de borda ou de borrifos (spray), e as duas seguintes na área de água corrente.
A primeira sessão se dá no topo da queda d'água e a última na parte baixa onde se forma a espuma da queda (entre 30 e $40 \mathrm{~cm}$ da linha d'água), entre uma e outra se estabelece outras quatros sessões equidistantes ao longo do paredão, no intervalo entre a primeira e última. Ao numerar as subunidades, considera-se a mais baixa (última a ser coletada) como número 1 e a do topo $\left(1^{\mathrm{a}}\right.$ coletada) como número 18 , mantendo assim o mesmo sentido de ordenação que se faz quando se coleta de jusante a montante em trechos sem cachoeiras (nas transecções lineares de 100m).

A metodologia aqui proposta foi testada em cachoeiras, com paredões variando de 12 a $25 \mathrm{~m}$ de altura, e funcionou adequadamente para coletas de larvas de EPT (Ephemeroptera, Plecoptera e Trichoptera), Diptera e Coleoptera, ninfas de Heteroptera e Odonata, bem como adultos de Heteroptera e Coleoptera.

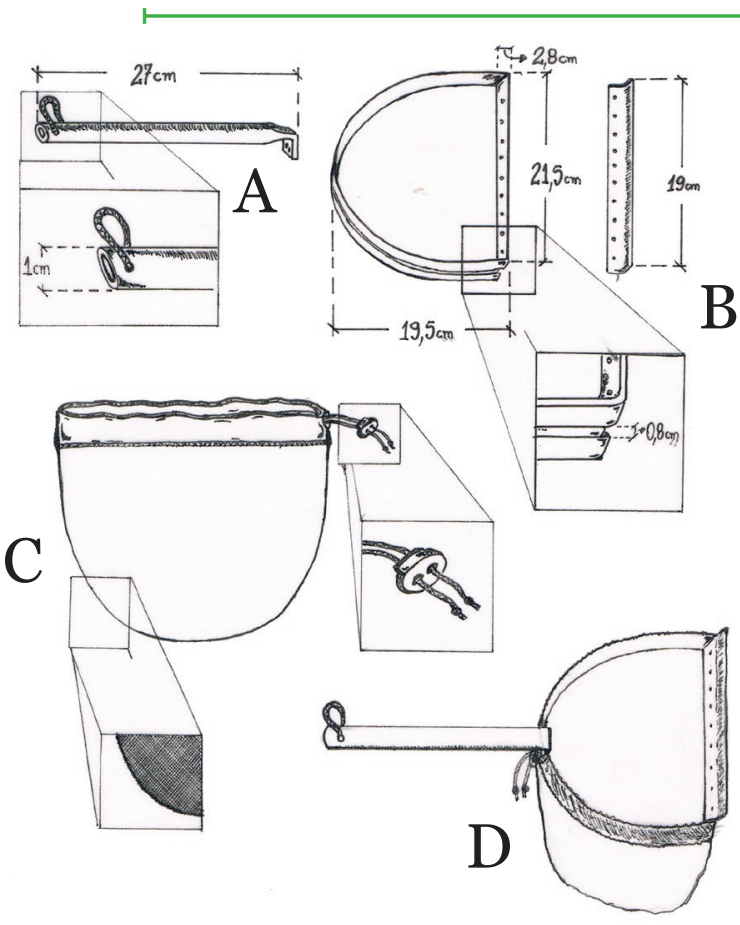

Figura 1. A - D. Coletor para insetos aquáticos em paredões de cachoeiras. Cabo (A), aro com detalhe de aba e ranhura (B), rede com detalhe da tela e presilha (C) e coletor completo (D).

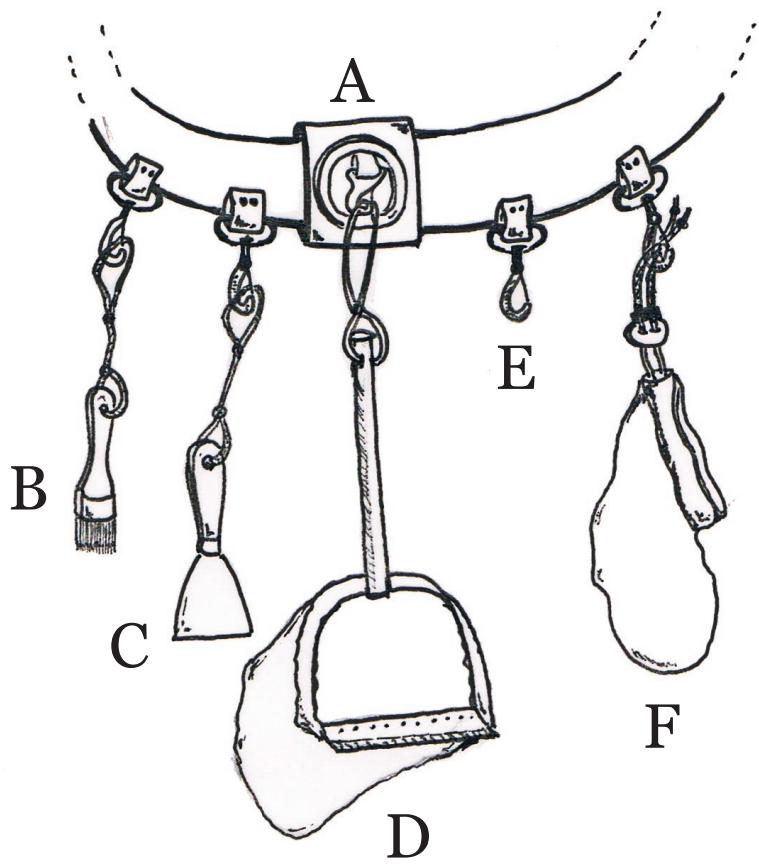

Figura 2. A - F. Cinturão para acomodar instrumentos de coleta de insetos aquáticos em cachoeiras. Reforço central (A), trincha (B), espátula de pedreiro (C), coletor completo (D), mini mosquetão (E) e rede sobressalente $(\mathrm{F})$.

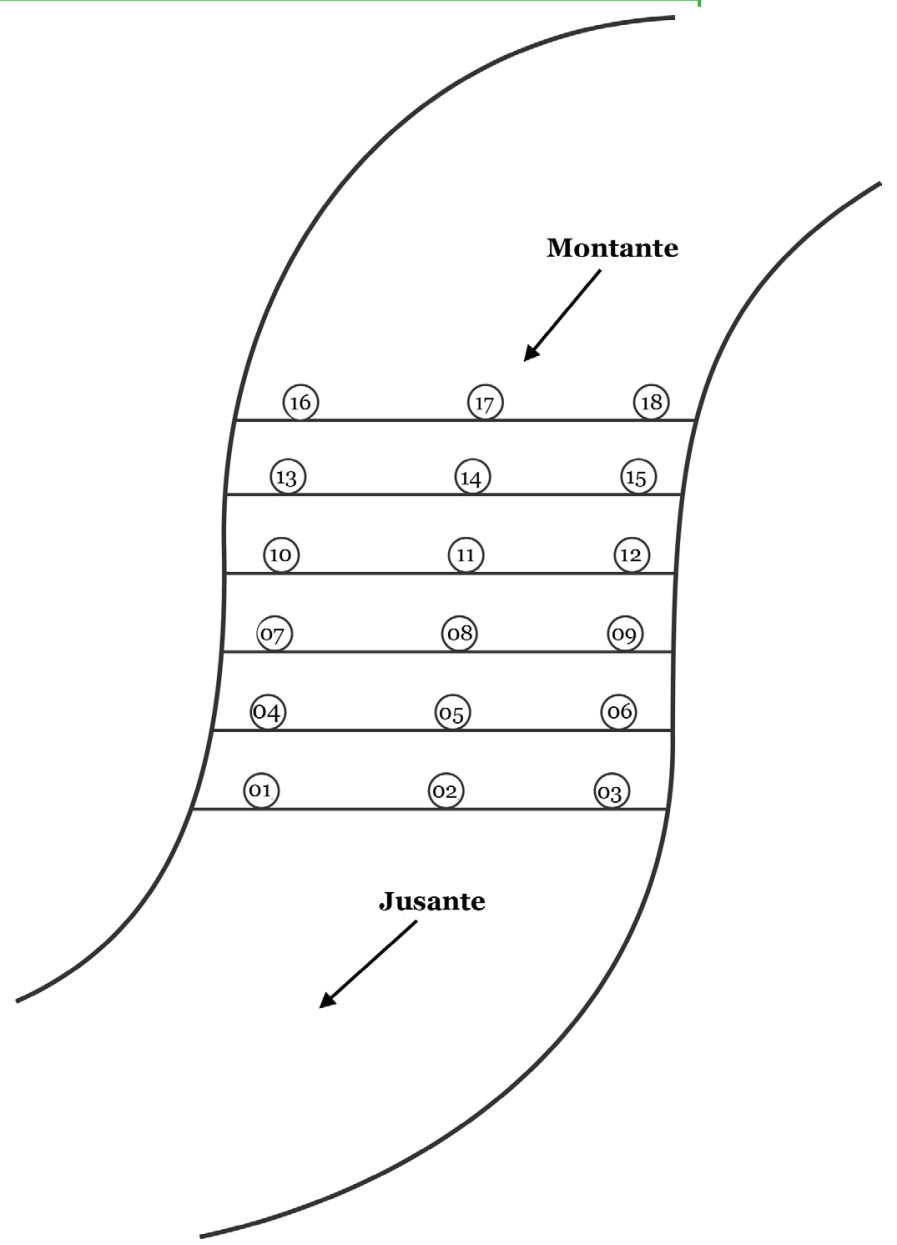

Figura 3. Modelo de transecção definida para coleta de insetos aquáticos em paredões de cachoeiras com alturas variáveis.

\section{AGRADECIMENTOS}

A FAPEMAT (proc. $\mathrm{n}^{\mathrm{o}} 465866 / 2009$ ) pelo fomento, aos proprietários e gerentes das fazendas que gentilmente nos deram acesso aos locais, a Anselmo Segatti que treinou a equipe de coleta em rapel nos paredões e Murilo Passarelli que desenhou a rede de captura.

\section{REFERÊNCIAS}

Cabette, H.S.R., N.F.S. Giehl, K. Dias-Silva, L. Juen \& J.D. Batista, 2010. Distribuição de Nepomorpha e Gerromorpha (Insecta: Heteroptera) da Bacia do Rio Suiá-Missu, MT: riqueza relacionada à qualidade de água e de hábitat, p. 113-137. In: Santos, J.E., C. Galbiati \& L.E. Moschini (Orgs.). Gestão e Educação Ambiental: Água, biodiversidade e cultura, v.2. São Carlos, RiMa, 409p.

De Marco, P.Jr., 1998. The Amazonian campina dragonfly assemblage: patterns in microhabitat use and behavior in a 
foraging habitat. Odonatologica, 27: 239-248.

Dias-Silva, K., H.S.R. Cabette, L. Juen \& P.Jr. De Marco, 2010. Habitat integrity and physical-chemical water variables influence on the structure of aquatic and semi-aquatic Heteroptera. Revista Brasileira de Zoologia, 27: 918-930.

Merritt, R.W., K.W. Cummins \& V.H. Resh, 1984. Collecting, sampling, and rearing methods for aquatic insects, p. 11-26. In: Merritt, R.W. \& K.W. Cummins (Eds.). An Introduction to the aquatic insects of North America, 2 ed. Dubuque, Kendall/Hunts, 311p.

Nessimian, J.L., E. M. Venticinque, J. Zuanon, P.Jr. De-Marco, M. Gordo, L. Fidelis, J.D. Batista \& L. Juen, 2008. Land use, habitat integrity and aquatic insect assemblages in Central Amazonian streams. Hydrobiologia, 614: 117-131.

Pereira, L.R., H.S.R. Cabette \& L. Juen, 2012. Trichoptera as bioindicators of habitat integrity in Pindaíba river basin, Mato Grosso (Central Brazil). Annales de Limnologie, 48: 295-302.
Roldán-Pérez, G., 1988. Guía para el estúdio de los macroinvertebrados acuáticos del Departamento de Antioquia, Universitad de Antioquia, 217p.

Silva-Pinto, N.L. Juen, H.S.R. Cabette \& P.Jr. De Marco, 2012. Fluctuating asymmetry and wing size of Argia tinctipennis Selys (Zygoptera: Coenagrionidae) in relation to riparian forest preservation status. Neotropical Entomology, 41: 178185 .

Souza, H.M.de, H.S.R. Cabette \& L. Juen, 2011. Baetidae (Insecta, Ephemeroptera) em córregos do cerrado matogrossense sob diferentes níveis de preservação ambiental. Iheringia, Série Zoologia, 101: 181-190.

\section{Recebido em: 05/04/2013}

Aceito em: 28/04/2014

\section{Como citar este artigo:}

Cabette, H.S.R. \& L.A. Castro, 2014. Metodologia de Coleta de Insetos Aquáticos em Paredões de Cachoeiras. EntomoBrasilis, 7 (2): $161-163$ Acessível em: doi:10.12741/ebrasilis.v7i2.339
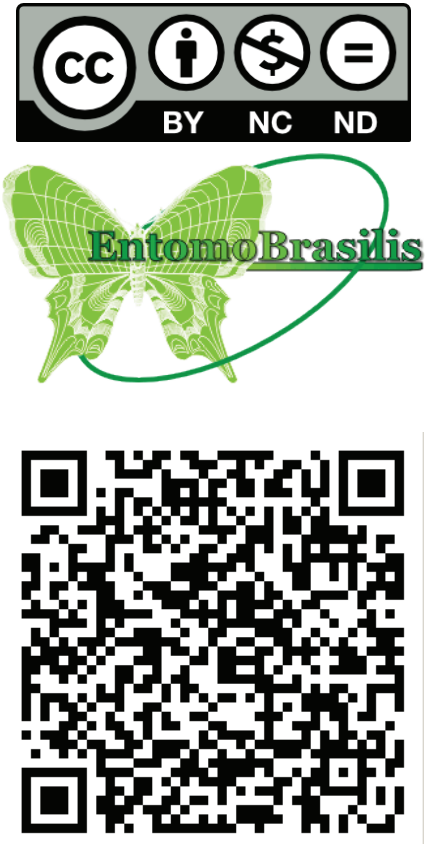